\title{
Mechanism of action of vesicular monoamine transporter 2 (VMAT2) inhibitors in tardive dyskinesia: reducing dopamine leads to less "go" and more "stop" from the motor striatum for robust therapeutic effects
}

\author{
Stephen M. Stahl
}

ISSUE:

Tardive dyskinesia can now be successfully treated by inhibiting the vesicular monoamine transporter type 2 (VMAT2).

\section{Take-Home Points}

- Inhibition of the vesicular monoamine transporter

type 2 (VMAT2) reduces dopamine storage and

\section{release.}

- Diminishing dopamine release in turn curtails the

hypothetical overstimulation of supersensitive D2 dopamine

receptors in the motor striatum that causes tardive

dyskinesia.

- Trimming dopamine release via VMAT2 inhibition in the motor striatum results in stronger "stop" signals and weaker "go," signals and thus robust therapeutic effects in reducing the abnormal involuntary hyperkinetic movements of tardive dyskinesia.

\section{Introduction}

Until recently, therapeutic options for tardive dyskinesia (TD) were few and unsatisfactory. The introduction of drugs with a new mechanism of action, namely inhibition of the vesicular monoamine transporter type 2 (VMAT2), is now transforming the treatment of TD. ${ }^{1,2}$ Here we review dopamine neurotransmission (see also References ${ }^{3-8}$ ) and how it is affected by TD and by various drugs to set the stage for the significance of this new breakthrough treatment mechanism.

\section{VMAT2 and Its Inhibition by Tetrabenazine}

Transporters for neurotransmitters exist not only on presynaptic nerve terminals (the well-known "reuptake pumps" targeted by most drugs for depression), but also on synaptic vesicles. ${ }^{3}$ Several types of vesicular transporters have been identified, including different ones for gamma amino butyric acid (GABA), glutamate, glycine, acetylcholine, monoamines, and others. ${ }^{3}$ The specific transporter known as VMAT2 is located on synaptic vesicles of dopamine, norepinephrine, serotonin, and histamine neurons to store these neurotransmitters until needed for release during neurotransmission. ${ }^{3-7}$ VMAT2 can also transport certain drugs as "false" substrates, such as amphetamine and ecstasy (MDMA; 3,4-methylenedioxymethamphetamine), which can compete for the "true," natural neurotransmitter. ${ }^{3}$ Synaptic vesicles create low $\mathrm{pH}$ in their lumens (interiors) with an energyrequiring proton pump there. ${ }^{3,6}$ Low $\mathrm{pH}$ in turn serves as the driving force to sequester the neurotransmitter in the synaptic vesicle. ${ }^{3,6}$

There are actually two types of VMATs: VMAT1, which is localized in both the peripheral and central nervous system (CNS), and VMAT2, located only in the CNS. $^{3-7}$ There are two known VMAT inhibitors: 


\section{BRAINSTORMS-Clinical Neuroscience Update}

Figure 1. Tardive dyskinesia as imbalance in "stop" and "go" signals from the motor striatum. D2 receptors in the indirect pathway of the motor striatum hypothetically react to chronic blockade by D2 antagonists by "learning" to have tardive dyskinesia with aberrant neuronal plasticity, resulting in supersensitivity to dopamine. This leads to too much inhibition of "stop" signals coming from too much dopamine acting at upregulated D2 receptors in the indirect pathway (on the right), and unopposed "go" signals coming from the direct pathway (on the left), and thus involuntary hyperkinetic movements.

Tardive Dyskinesia : Supersensitive D2 Receptors

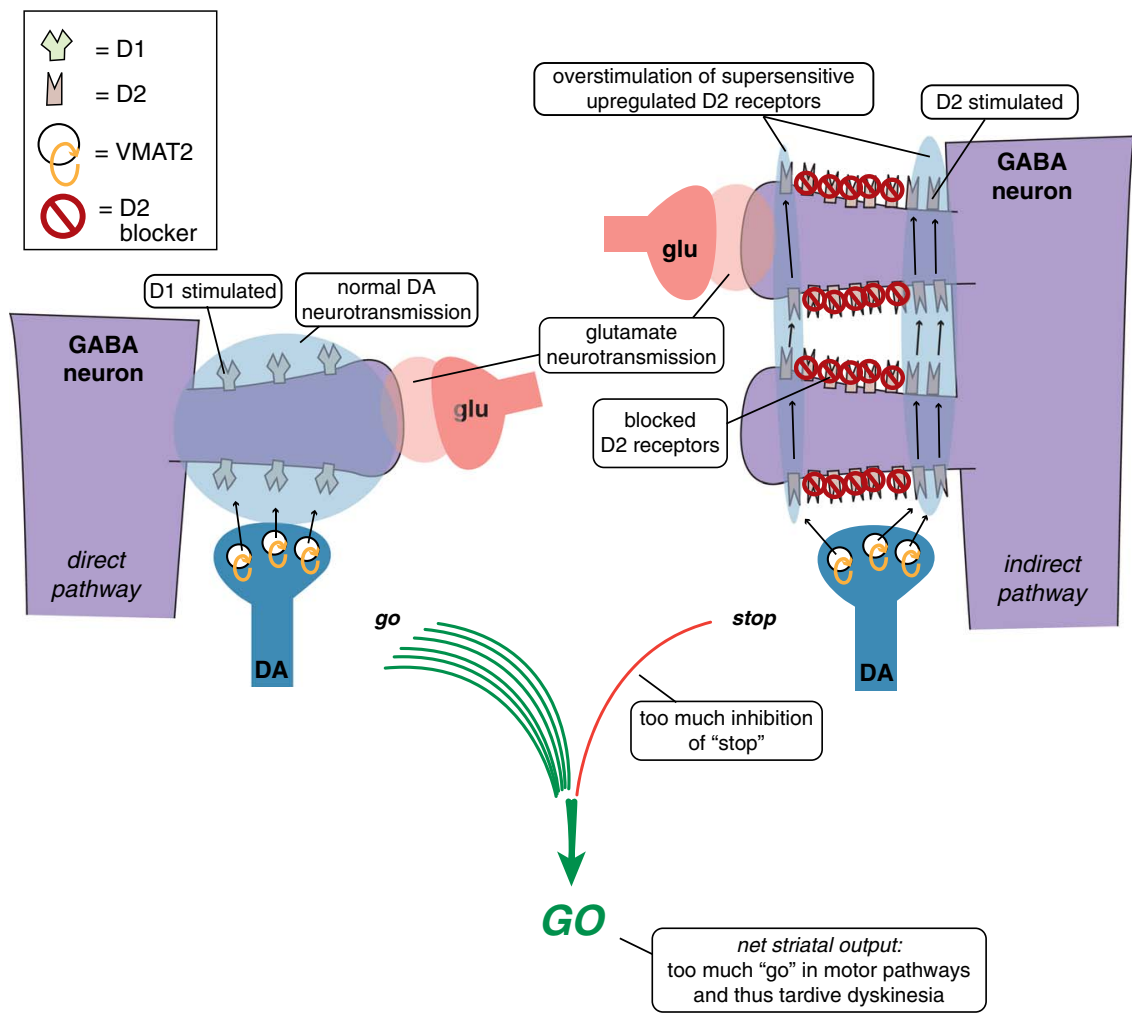

reserpine, which inhibits irreversibly both VMAT1 and VMAT2, and tetrabenazine, which reversibly inhibits only VMAT2. That is why reserpine-related drugs, but not tetrabenazine-related drugs, are associated with frequent peripheral side effects, such as orthostatic hypotension (reserpine was once used for hypertension), stuffy nose, itching, and gastrointestinal side effects. Although VMAT2 transports multiple neurotransmitters into synaptic vesicles, tetrabenazine preferentially affects dopamine at clinical doses. ${ }^{7}$ When tetrabenazine blocks the transport of dopamine into presynaptic vesicles, dopamine is rapidly degraded by monoamine oxidase (MAO), leading to depletion of presynaptic dopamine proportionate to the degree of VMAT2 inhibition. ${ }^{3}$ Tetrabenazine itself is rapidly absorbed and converted into active metabolites, especially alpha and beta hydroxytetrabenazine. ${ }^{7}$ Deuterated tetrabenazine is similarly metabolized, but more slowly; valbenazine is converted only into the alphahydroxy metabolite. ${ }^{1,2,7}$

\section{Treating Overstimulation of D2 Receptors in Tardive Dyskinesia}

D2 receptors in the indirect pathway of the motor striatum hypothetically react to chronic blockade by D2 antagonists by "learning" to have tardive dyskinesia with aberrant neuronal plasticity, resulting in supersensitivity to dopamine. ${ }^{4}$ This leads to too much inhibition of "stop" signals coming from too much dopamine acting at upregulated D2 receptors in the indirect pathway (Figure 1 on the right) and unopposed "go" signals coming from the direct pathway (Figure 1 on the left), and thus involuntary hyperkinetic movements. ${ }^{4}$ If the brain has literally "learned" to have TD in 


\section{BRAINSTORMS —Clinical Neuroscience Update}

Figure 2. Raising the dose of D2 blockers in tardive dyskinesia. In an attempt to suppress unwanted hyperkinetic movements of TD, one can try to raise the dose of D2 antagonist to block some of those upregulated supersensitive D2 receptors. This

might work short term in some patients but at the expense of more immediate side effects and the prospects of making TD even worse long term.

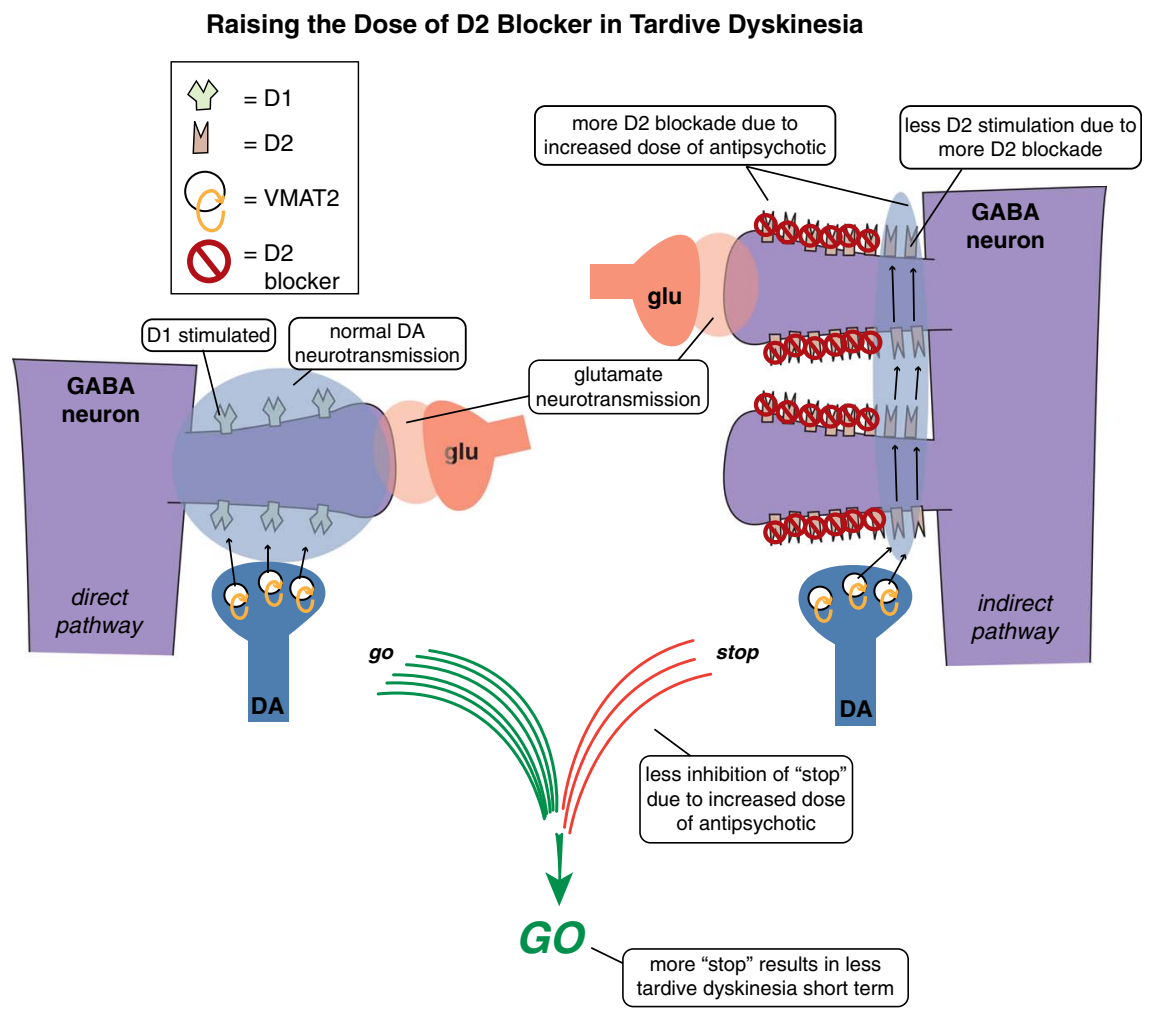

an aberrant attempt to compensate for chronic D2 blockade that results in dopamine overstimulation, then TD would seem to be a disorder ideally set up to respond to interventions that lower dopamine neurotransmission. How can this be done?

One way is to raise the dose of D2 antagonist to block those upregulated supersensitive D2 receptors (Figure 2). This might work in the short term in some patients at the expense of more immediate side effects and the prospects of making TD even worse down the road. ${ }^{9}$ Another treatment possibility is to stop the offending D2 antagonist with the hope that the motor system will re-adjust back to normal and the movement disorder will reverse (Figure 3). Indeed, many patients who do not have an underlying psychotic disorder may be able to tolerate the discontinuation of their D2 antagonist. Unfortunately, it does not seem that the TD brain can "forget" its aberrant neuroplastic learning very well, and only some patients-particularly those who discontinue D2 blockade soon after the onset of their movements-will enjoy reversal of their TD. ${ }^{9}$ In fact, most patients experience an immediate worsening of their movements when D2 blockade is eliminated, due to the completely unblocked actions of dopamine in the absence of any D2 antagonist therapy at all (Figure 3).

VMAT2 inhibition is a mechanism that reduces dopamine stimulation without blocking D2 receptors. Thus, this action reduces the overstimulation of D2 receptors in the indirect pathway (on the right in Figure 4), resulting in less inhibition of the stop signal there. However, there is also a benefit of VMAT2 inhibition in the direct pathway where "go" signals are amplified by dopamine at D1 receptors. ${ }^{4,5}$ Even though these D1 receptors and this direct extrapyramidal pathway may not be the site of pathology in TD, ${ }^{4}$ they do drive "go" signals for movement, 6 and lowering dopamine there by VMAT2 inhibition would thus be expected to lower the "go" signals arising from the direct pathway (Figure 4 on the left). ${ }^{4}$ Combined with 


\section{BRAINSTORMS-Clinical Neuroscience Update}

Figure 3. Discontinuing D2 blockers in tardive dyskinesia. Sometimes it is necessary or desirable to discontinue the D2 antagonist causing TD in the hope that the motor system will re-adjust back to normal and the movement disorder will reverse. Sometimes the underlying disorder will not allow that. However, if it is possible to discontinue D2 antagonist treatment, it is unfortunately uncommon that the movement disorder reverses. In fact, most patients experience an immediate worsening of their movements when D2 blockade is eliminated, due to the completely unblocked actions of dopamine in the absence of any D2 antagonist therapy at all.

\section{Discontinue D2 Blocker in Tardive Dyskinesia}

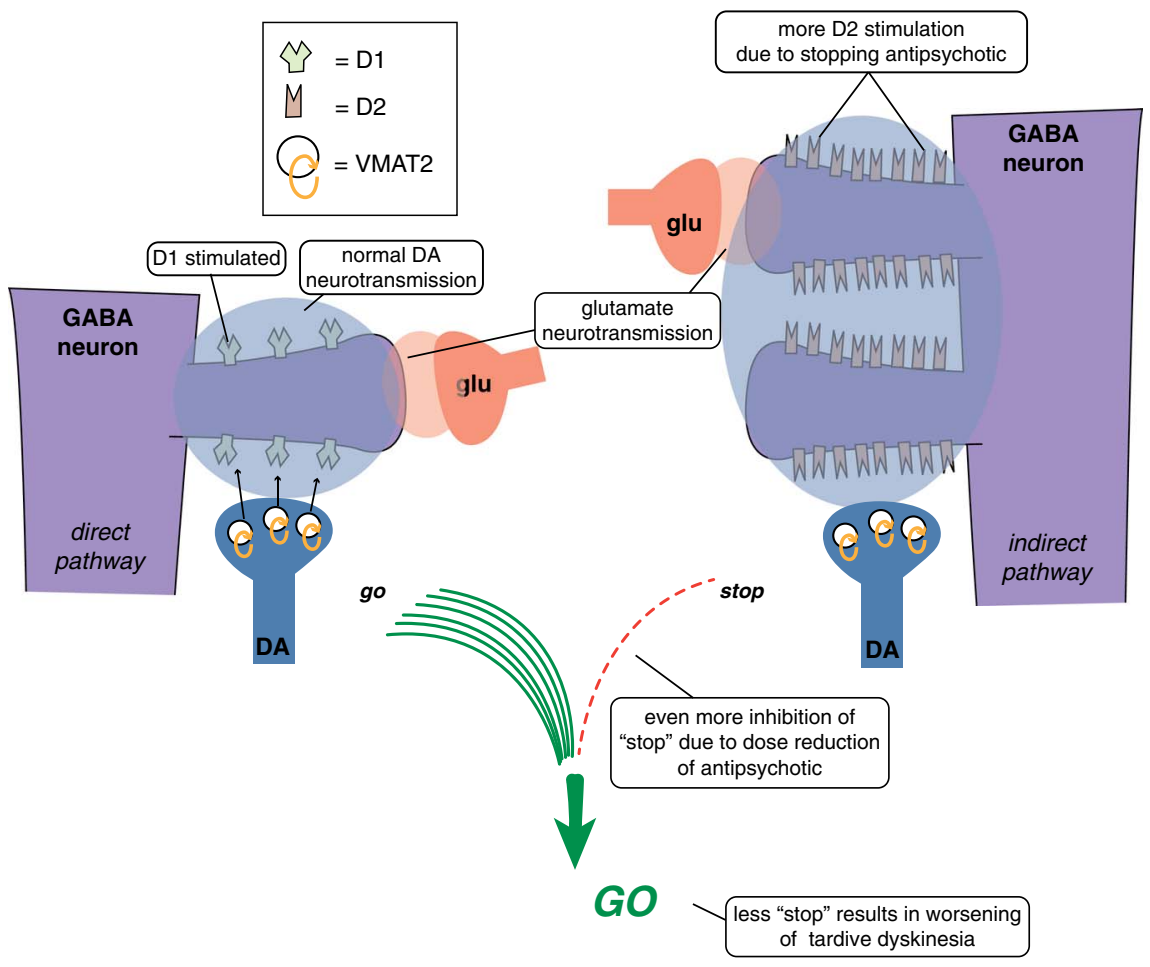

more "stop" signals from the indirect pathway (Figure 4 on the right), motor output to drive abnormal involuntary hyperkinetic movements is therefore robustly reduced by this combination of effects of dopamine depletion in both pathways.

So, it appears that VMAT2 inhibition can "train" motor pathways of TD to compensate for their abnormal "learning" after chronic D2 receptor blockade. ${ }^{1,2,4,7}$ Whether this will be disease modifying in the long run, and whether it will reverse rather than only treat movements symptomatically, must be determined by long-term studies of VMAT2 inhibition in TD.

\section{Variable in Treating TD with VMAT2 Inhibition}

Treating TD is not going to be simple (Figure 5). That is, there will be a balancing act between the need to continue D2 blockade, which caused TD in the first place (Figure 1), to increase its dose (Figure 2), or whether the D2 blocker can be discontinued (Figure 3). All these choices will affect the impact of dopamine stimulation on the symptoms of TD differently, and thus will affect the dose of VMAT2 inhibitor and degree of VMAT2 inhibition needed in a given patient.

However, even this is not the whole story. Since VMAT2 inhibition is reversible, the amount of inhibition in a given patient at a given dose will be dependent upon the concentration of dopamine in the presynaptic neuron (Figure 5). ${ }^{3}$ Dopamine levels can be affected by drugs (such as amphetamine and MAO inhibitors) and disease state (such as stress, psychosis, and depression). ${ }^{3}$ The degree of VMAT2 inhibition required for a given patient also depends upon several variables on the postsynaptic neurons receiving dopamine stimulation (Figure 5). That is, the number and sensitivity (and possible reversibility) of supersensitive D2 receptors will vary from one patient to another, with time, and in 


\section{BRAINSTORMS-Clinical Neuroscience Update}

Figure 4. VMAT2 inhibition in TD. This mechanism reduces dopamine stimulation without blocking D2 receptors. It will reduce overstimulation of D2 receptors in the indirect pathway (on the right), resulting in less inhibition of the stop signal there. It will also inhibit VMAT2 and reduce dopamine release in the direct pathway where "go" signals are amplified by dopamine at D1 receptors (on the left). Even though these D1 receptors and this direct extrapyramidal pathway may not be the site of pathology in TD, this pathway does drive "go" signals for movement, so lowering dopamine there by VMAT2 inhibition lowers "go" signals arising from the direct pathway (on the left). Combined with more "stop" signals from the indirect pathway (on the right), motor output to drive abnormal involuntary hyperkinetic movements can be robustly reduced by this combination of effects of dopamine depletion in both pathways.

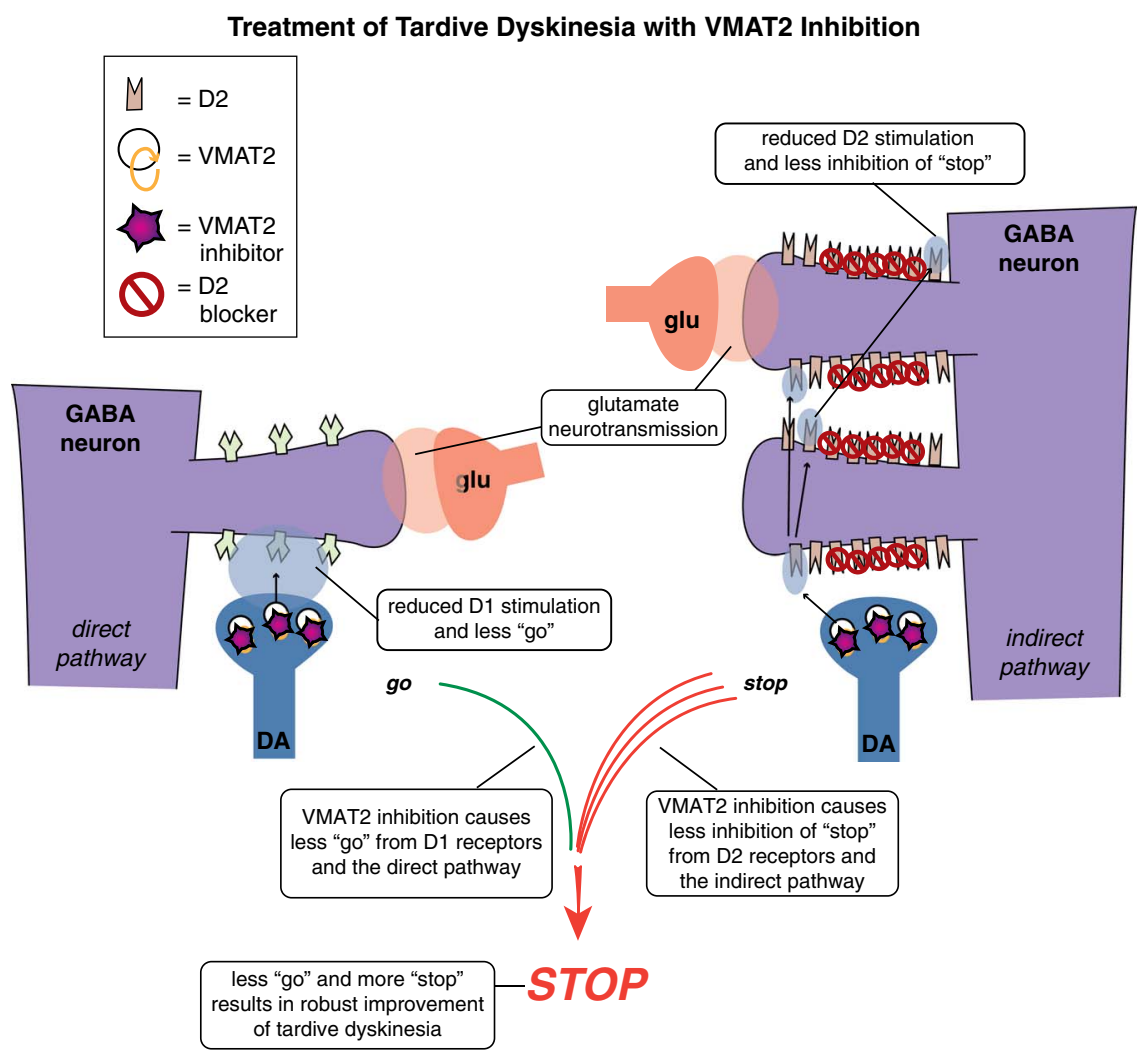

different areas of the motor striatum, depending upon which body parts are most affected. The degree of dopamine reduction caused by VMAT2 inhibition that is needed by a given patient will also vary with which specific D2 blocker is given, ${ }^{8}$ and at which dose, and with how much dopamine is being released into the synapse. Changes in psychosis status, mood, and other factors will change dopamine levels in the synapse and therefore the amount of VMAT2 inhibition required. That is, dopamine can compete with any D2 blocker administered, but this depends quite widely on which blocker is given (affinities for the D2 receptors have a broad range), and at which dose. ${ }^{8}$ Thus, the skilled clinician must balance these factors when administering a VMAT2 inhibitor to attain best results in the individual patient. When done well, there are very good prospects for robust improvements in TD in many patients.

\section{References:}

1. Hauser RA, Factor SA, Marder SR, et al. KINECT 3: a phase 3 randomized, double-blind, placebo-controlled trial of valbenazine for tardive dyskinesia. Am J Psychiatry. 2017; 174(5): 476-484.

2. Fernandez HH, Factor SA, Hauser RA, et al. Randomized controlled trial of deutetrabenazine for tardive dyskinesia. Neurology. 2017; 88(21): 2003-2010.

3. Stahl SM. Stahl's Essential Psychopharmacology: Neuroscientific Basis and Practical Application, 4th ed. Cambridge, UK: Cambridge University Press; 2013.

4. Stahl SM. Neuronal traffic signals in tardive dyskinesia: not enough "stop" in the motor striatum. CNS Spectr. 2017; 22(6): 427-434.

5. Stahl SM. Dazzled by the dominions of dopamine: clinical roles of D3, D2, and D1 receptors. CNS Spectr. 2017; 22(4): 305-311. 


\section{BRAINSTORMS-Clinical Neuroscience Update}

Figure 5. Variables in treating TD with VMAT2 inhibition. Treating TD with VMAT2 inhibitors requires balancing many issues: whether D2 blockade can be increased, decreased, or switched to another D2 blocker. All these choices will affect the impact of dopamine stimulation on the symptoms of TD differently, and thus will affect the dose of VMAT2 inhibitor and degree of VMAT2 inhibition needed in a given patient. In addition, VMAT2 inhibition is reversible, so the amount of inhibition in a given patient at a given dose will be dependent upon the concentration of dopamine in the presynaptic neuron, which can be affected by drugs (such as amphetamine and MAO inhibitors) and disease state (such as stress, psychosis, depression). The amount of VMAT2 inhibition and reduction of dopamine stimulation required for a given patient also depends upon several variables on the postsynaptic neurons receiving dopamine stimulation. That is, the number and sensitivity (and possible reversibility) of supersensitive D2 receptors will vary from one patient to another, with time, and in different areas of the motor striatum, depending upon which body parts are most affected. The degree of dopamine reduction caused by VMAT2 inhibition that is needed by a given patient will also vary with which specific D2 blocker is given, at which dose, and with how much dopamine is being released into the synapse. Changes in psychosis status, mood, and other factors will change dopamine levels in the synapse and therefore the amount of VMAT2 inhibition required. Since dopamine can compete with any D2 blocker, the effects of any D2 blocker depend upon the affinity of the drug for the D2 receptor and the dose of drug.

Variables Controlling Output from the Indirect Pathway Via D2 Receptors

$M=$ D2
$=$ VMAT2
$=$ = VA
$=$ inhibitor
blocker
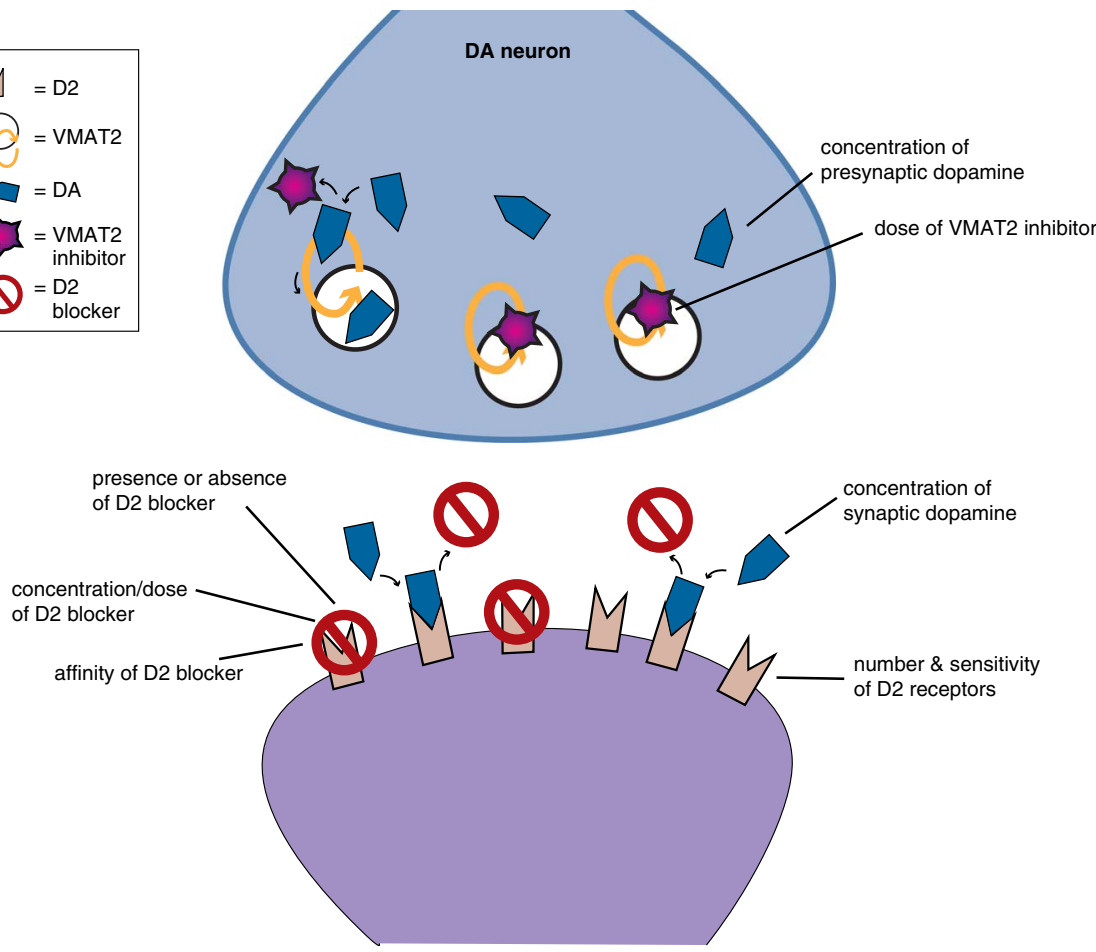

6. Lawal HO, Krantz DE. SLC18: vesicular neurotransmitter transporters for monoamines and acetylcholine. Mol Aspects Med. 2013; 34(2-3): 360-372.

7. Jankovic J. Dopamine depleters in the treatment of hyperkinetic movement disorders. Expert Opin Pharmacother. 2016; 17(18): 2461-2470.
8. Stahl SM. Drugs for psychosis and mood: unique actions at D3, D2, and D1 dopamine receptor subtypes. CNS Spectr. 2017; 22(5): 375-384.

9. Caroff SN, Campbell EC. Drug-induced extrapyramidal syndromes: implications for contemporary practice. Psychiatr Clin North Am. 2016; 39(3): 391-411. 